\title{
Emergence of Social Rumor: Modeling, Analysis, and Simulations
}

\author{
ZhengYou Xia and LaiLei Huang \\ Department of computer science, Nanjing University of Aeronautics and Astronautics \\ Zhengyou_xia@yahoo.com
}

\begin{abstract}
We define the notion of social rumor in a standard game-theoretic framework, and assume each agent in the rumor game with individual rationality. In this framework, individual agent can interact with its neighboring agents, and word-of-mouth communication is employed during interaction. We introduce a simple and natural strategy-select rule, called behavior update rule (BUR). The BUR uses an accumulative influence force (CIF) with considering the authority influence of neighboring agents rather than simple accumulative number of information from neighboring agents. The BUR can provide rules to restrict agents' behavior to one particular strategy and lead to emergence of social rumor or anti-rumor. Most importantly, we give simple natural rules of rumor and anti-rumor information transmission, and investigate the efficiency with which social rumors and anti-rumors (agent claims that rumor information is false or doesn't exist) are achieved.
\end{abstract}

\section{Introduction}

Emergent behavior is a key topic in artificial life research, given that artificial life typically adopts a bottom-up approach to modeling various forms of collective behavior, belief and emergent social phenomena that are observed in the societies ${ }^{[1][8]}$. Society is regarded as emerging from interactions among individuals (agents). The action of an agent is social if it is performed towards another agent with purpose, considering the other agent also as a purposeful entity. To model social action it is necessary to go beyond the individual (single agent) level of analysis to reach a multiagent notion of social intelligence. A more general problem is posed by the simulation studies on social dynamics, namely how given social patterns emerge from autonomous agents in a common artificial world ${ }^{[2][3][4]}$.

Rumors are social properties and part of our everyday life. Rumors can shape the public opinion of a society by affecting and coordinating the individual beliefs of its members. Related researches about rumors include work in economics, sociology and psychology, etc. Economists ${ }^{[5]}$ have looked at rumors, both from a theoretical and an empirical point of view that are focused on rumor, price and market selection. In the sociology, psychology, and policy management domain, researches about rumors are focused mainly on the effect of rumors on management, policymaking, the role of uncertainty, anxiety, and psychology of rumors ${ }^{[5,6]}$. Recent researches about rumor 
propagation have been achieved by Zanette ${ }^{[7][8] .}$ In their paper, epidemiological SIR model is employed and each element of $\mathrm{N}$-element population adopts one of three possible states. To use epidemiological SIR model cannot well model some properties of social rumor. For example, how to model social rumor and anti-rumor that are coexistent in the same social network? How to model the evolution of each agent' belief about rumor (or anti-rumor) and the strategies of each agent in the rumor or anti-rumor environment? In other words, previous model ${ }^{[7][8]}$ is focused on rumor propagation mechanism and cannot validly deal with the evolution about belief (or strategy) of each agent in the coexistence of rumor and anti-rumor environment.

Addressing the above problems, we present a simple model and some rules to discuss the emergence of social rumor and anti-rumor by word-of-mouth communication. We first model social rumor information by using game theory, and then discuss the emergence of social rumor and anti-rumor based on multi-agent systems. Roughly speaking, this process we aim to study is one in which individual agent interacts with one another, and information is transmitted with word-of-mouth communication. Based on its personal accumulated information from other neighboring agents, each agent updates its belief about information over time.

\section{Games, Social Rumors and Agents}

In this section, we lay out the framework, starting with the standard game-theoretic notion, and overlaying those with the notions of social rumors and agent.

\subsection{Games}

Game theory ${ }^{[9]}$ is a branch of mathematical analysis developed to study decision making in conflict situations. A game involves a number of players, each of which has available to it a number of possible strategies. Depending on the strategies selected by each player, they each receive a certain payoff. As rational players, they always try to maximize their payoff. In this article, we adopt without change the notions of games, payoff matrices, and rationality as utility maximization that are described in the game theory.

Rumors are part of our everyday life, e.g. "price of butter will run up in one month". Related definitions about rumor have been described by different papers ${ }^{[5][6]}$. We adopt the standard definition and focus it on the multi-agent systems environment.

Definition 1. Rumor Information (RI): A piece of news (or information) that is passed from agent to agent through word-of-mouth communication and which may or may not be true.

In this article, we assume the initiator of rumor information will get some payoff (money or spirit satisfaction) when he generates rumors. The content of rumor information is assumed to affect the benefit of agents (money or spirit). Therefore, the initiator of rumor and individual (agent) that he hears this rumor will form a twoperson general-sum game. 
Definition 2. Rumor Game: The normal form of a rumor game is as follow.

$$
\left.\begin{array}{cccc}
\multicolumn{2}{c}{\text { Agent }} \\
\text { Initiator } & \text { rumor(true }) & \text { S } 2 \\
\text { rumor }(\text { false }) & (\text { true }, S 1), B(\text { true }, S 1) & A(\text { true }, S 2), B(\text { true }, S 2) \\
\text { A(false }, S 1), B(\text { false }, S 1) & A(\text { false }, S 2), B(\text { false }, S 2)
\end{array}\right)
$$

Where, S1 and S2 is denoted as strategy 1 and strategy 2 of agent, respectively. A and $\mathrm{B}$ is denoted as real -value function of initiator and agent, respectively.

In the rumor game, initiator has two kinds of strategies, which is information that he has generated is true and false. We suppose individual (agent) has two strategies that are S1 or S2 when agent receives rumor information. We suppose that agent is rational and rumor game has no dominated strategy for agents. When an agent believes that rumor information is true, It will use one strategy to maximize itself payoff (i.e., $\max \{B($ true,$S 1), B($ true,$S 2)\})$. When the agent doesn't believe that rumor information (i.e., agent believe that information is false), he will choose one strategy to maximize itself payoff (i.e., $\max \{B($ false,$S 1), B($ false,$S 2)\}$ ). When belief of agent for rumor information is uncertainty, agent will use mixed strategies to play rumor game.

\subsection{Agent and Emergence of Social Rumors}

In human societies, each agent has its social authority (or position). Generally, an agent with low authority (position) may be liable to believe information said by the agent with high authority (position). In other words, when agent $i$ transmits information to agent $j$, the belief of agent $j$ about this information is affected by the social authority of agent $i$.

Definition 3. Social Authority of agent $i$ can be: $A_{i} \in[0, \phi]$, where $\phi$ is a natural number.

$A_{i}>A_{i}$ means that the authority of agent $j$ is superior to agent $i$. Suppose agent $i$ transmits information to agent $j$, How to measure the influence force that social authority of agent $i$ affects the belief of agent $j$ about this information?

Definition 4. Influence Force ( $I F$ ): Belief of the agent $i$ affected by social authority of neighboring agent $j$ can be a function: $I F_{i j}=I n f \_t y p e * e^{k\left(A_{,}-A_{i}\right)}$,

Where, $A_{i}, A_{j}$ is social authority of agent $i$ and agent $j$, respectively. $k$ is coefficient and $k \in[0,1] . k$ is denoted as the degree that agent $i$ ratifies authority. If $\mathrm{k}=1$, it means that agent $i$ ratifies fully authority of social agent. Similarly, if $\mathrm{k}=0$, it means that agent $i$ doesn't ratify authority of social agent, in other words, the agent $i$ thinks that its social authority is equal to agent $j$ 's. In simulation experiment of this paper, the default value of $k$ is 0.5 . Inf_type is type of information received by agent $i$. We assume two kinds of information types (Inf_type) that are rumor and anti-rumor 
information. In this paper, we use " +1 " and " -1 " to denote as type of the rumor and anti-rumor information, respectively.

In the multi agent systems environment, agent $i$ will move and interact with $m$ different neighboring agents, which transmit information to agent $i$. Cumulative Influence Force (CIF) of agent $i$ affected by $m$ different neighboring agents can be computed by the following equation.

$$
C I F=\sum_{j} I n f-t y p e * e^{k\left(A_{,}-A_{i}\right)}
$$

where, $j$ be $m$ different neighboring agents

In human societies, when an agent $i$ receives information from agent $j$, the agent $i$ may not believe this information. However, when agent $i$ receives enough more same information from different neighboring agents, he will believe fully this information. Evolution of belief about information can be described by Belief function ( $\Psi$ ) of agent $i$.

$$
\Psi(\text { information })=\left\{\begin{array}{lr}
\mathbf{C I F} / \vartheta & \mathbf{0} \leq|\mathbf{C} \mathbf{I F}|<\vartheta \\
\mathbf{1} & |\mathbf{C} \mathbf{I F}|>\vartheta
\end{array}\right\}
$$

Where, $\vartheta$ is the threshold of agent $i$ and each agent may have different thresholds.

In the above equation 2, If the value of $|\mathbf{C I F}|$ is equal or bigger than the threshold of agent (i.e., $\Psi($ information $)=1$ ) and CIF is negative, agent fully believes the antirumor information and selects one strategy to maximize itself payoff. If the value of $|\mathrm{CIF}|$ is equal or bigger than threshold of agent (i.e., $\Psi($ information $)=1)$ and CIF is positive, agent fully believes the rumor information and selects one strategy to maximize itself payoff. If $|\mathrm{CIF}|$ is less than the threshold of the agent, the agent will use mixed strategies because the agent is uncertain about rumor or (and) anti-rumor information.

Definition 5. [Emergence of Social Rumor]: A belief about rumor information that restricts the agents' behavior to one particular strategy is called emergence of social rumor.

\section{Behavior Update Rules}

Before we discuss behavior update rule, we assume that each agent has following properties.1). Each agent is rational, which means that each agent will select one strategy to maximize itself payoff.2). Each agent can move randomly in some scope. Each agent moves and interacts with neighboring agents.3). Each agent uses wordof-mouth as communication method with neighboring agents.

We classify agents into three types, which are garrulous agent, close-lipped agent, and dumb agent. Although garrulous agent doesn't know whether information is true or not, it always likes to propagate information to their neighboring agents. The closelipped agent is different to garrulous agent. The close-lipped agent says something that he will believe and doesn't say something that he doesn't believe. The dumb 
agent doesn't say anything to others agent in any case. In this paper, we omit to consider dumb agent because the number of the dumb agent is very few in practical society

When rumor information is spread among agents, due to the lack of evidence that is typically involved in the transmission of a rumor (agents don't know whether the rumor is true or not), the probability with which an agent believes whether a rumor is true depends on the number of neighbors that communicate the same rumor information (it is not anti-rumor information). Intuitively, if you hear a story once, you may believe it or not, but if you hear it also from several persons with the same story, you become to believe that the story is true. Therefore, when every neighboring agent communicates rumor information with an agent, the probability that the agent becomes to believe the rumor increases. We use the notion of Cumulative Influence Force (CIF) to define behavior the update rule as following.

Definition 6. [Behavior Update Rule (BUR)]. When an agent hears information from neighboring agents, the agent believes fully the information and selects one new strategy iff absolute value of cumulative influencing force (CIF) of the agent about the information is equal or bigger than the threshold of the agent.

Behavior Update Rule (BUR) is a simple, natural and local update rule. In particular, it would be natural to consider update rule that uses an accumulation with considering authority influence of neighboring agents rather than simple accumulative number of information from neighboring agents.

\section{Experimental Results}

We describe a series of experiments in which we investigate the emergence of social rumor and anti-rumor based on multi-agent systems environment. 50 X 50 Grid and 200 agents are composed of simulation society and some agents are distributed randomly in the grid. Each agent can move random one step each time in the grid. Value of the threshold and authority of each agent in 50 X 50 grids are generated randomly.

\section{1) Emergence of social rumor and anti-rumor}

Fig1 shows comparison between the speed of emergence of social rumor and anti-rumor in the sparse environment. At the same experiment parameters, rumor and anti-rumor is emerged at about 171 and 65502 times of Iterations, respectively. Fig1 illustrates the practical social phenomenon, which is that rumor is easily emerged and anti-rumor is very difficult emerged. In the Figla, in the initial and middle phase, the curve is sleekly exponential increase and curve surges at final phase. The phenomenon can be explained by the following reasons. Agent will spend some time meeting its neighboring agents and it is difficult to make agents with big threshold and high authority to believe immediately rumor information. Although the above phenomenon exists, number of agents that believe rumor information mustn't decrease with increase of times of iterations in the rumor environment.

In the coexistence of rumor and anti-rumor environment, one agent may change belief from believing rumor to disbelieving rumor (believe anti-rumor) at one time when it hears anti-rumor information from neighboring agents. However, when the agent moves to another place, it may change from believing anti-rumor information to believing 
rumor information at this time when neighboring agents of this agent are all to believe rumor information. Therefore, number of agents that believe anti-rumor information may be decrease with increase of times of iterations at one time. The above phenomenon also often happens in the practical society. Figlb show the process of emergence of social anti-rumor and verifies above practical social phenomenon.
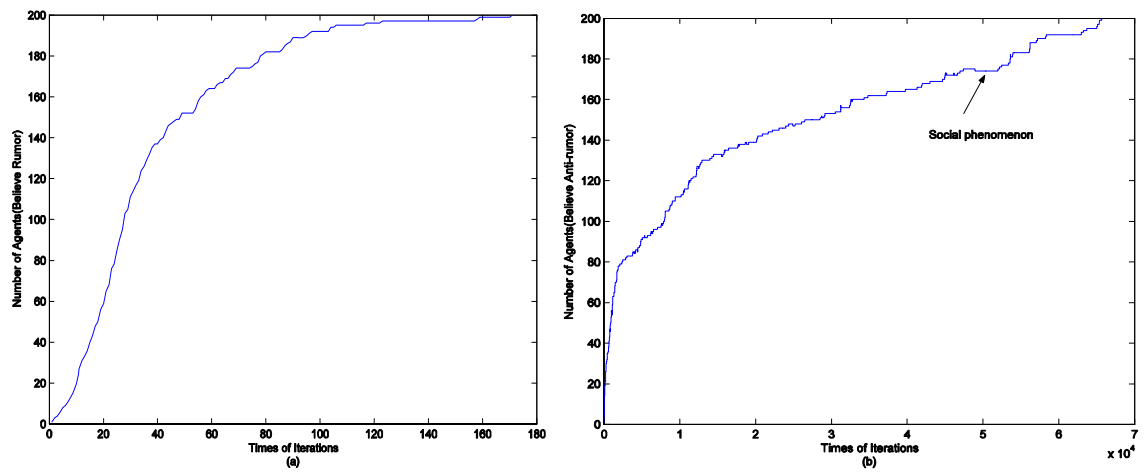

Fig. 1. Emergence of social rumor and anti-rumor :(a)Rumor. (b)Anti-Rumor

2) State of agent under coexistence of rumor and anti-rumor environment

When rumor and anti-rumor information are propagated together in practical society, there is one of three states for each person, which is to believe rumor, to disbelieve (to believe anti-rumor) and be uncertain for rumor and anti-rumor. Fig2 shows states of agents at 15000 times of iterations.

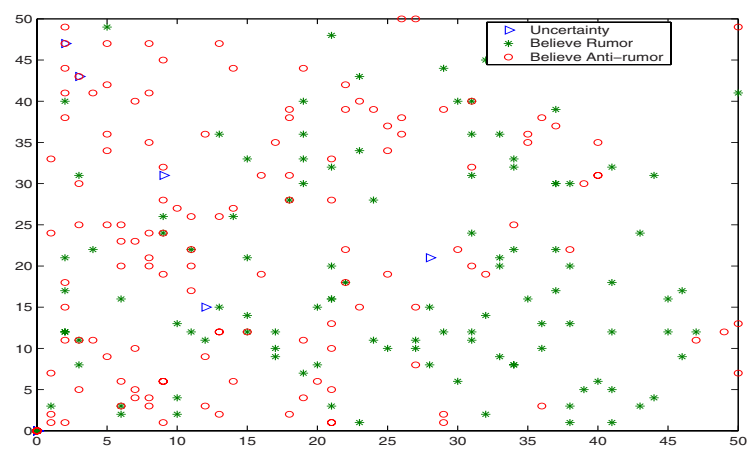

Fig. 2. State of agent

\section{3) Belief evolution of agent}

Let $p$ be in $(0,1)$ and $n$ be in $(-1,0) . n$ and $p$ are all denoted as uncertain belief of agent about information. In the rumor environment, evolution of agent's belief about rumor information is simple from 0 to 1 . Evolution paths of agent' belief only include two paths, which are $0 \rightarrow 1$ and , $0 \rightarrow p \rightarrow 1$. The above phenomenon is 
showed as Fig3a. However, in the coexistence of rumor and anti-rumor environment, Evolution of agent' belief about information is different to that in the rumor environment and is more complex(see Fig3b). Typical evolution paths of agent belief about information are as follows:

1. $1 \rightarrow-1$ : When an agent that fully believes rumor information hears anti-rumor information from neighboring agent(s), the agent change its behavior to full believe anti-rumor information.

2. $1 \rightarrow n \rightarrow-1$ : An agent full believes rumor information at one time. When the agent moves to one place and hears anti-rumor information from neighboring agent(s) at period of time, it will be in the uncertain belief for rumor and antirumor information. With increase of neighboring agents that believe anti-rumor information, the agent finally fully believes anti-rumor information.

3. $1 \rightarrow p \rightarrow-1$ : The evolution path of agent belief is similar to the second evolution path

4. $1 \rightarrow p \rightarrow n \rightarrow-1$ : The fourth evolution path is similar to the second and third evolution paths and evolves slowly than the above two paths.
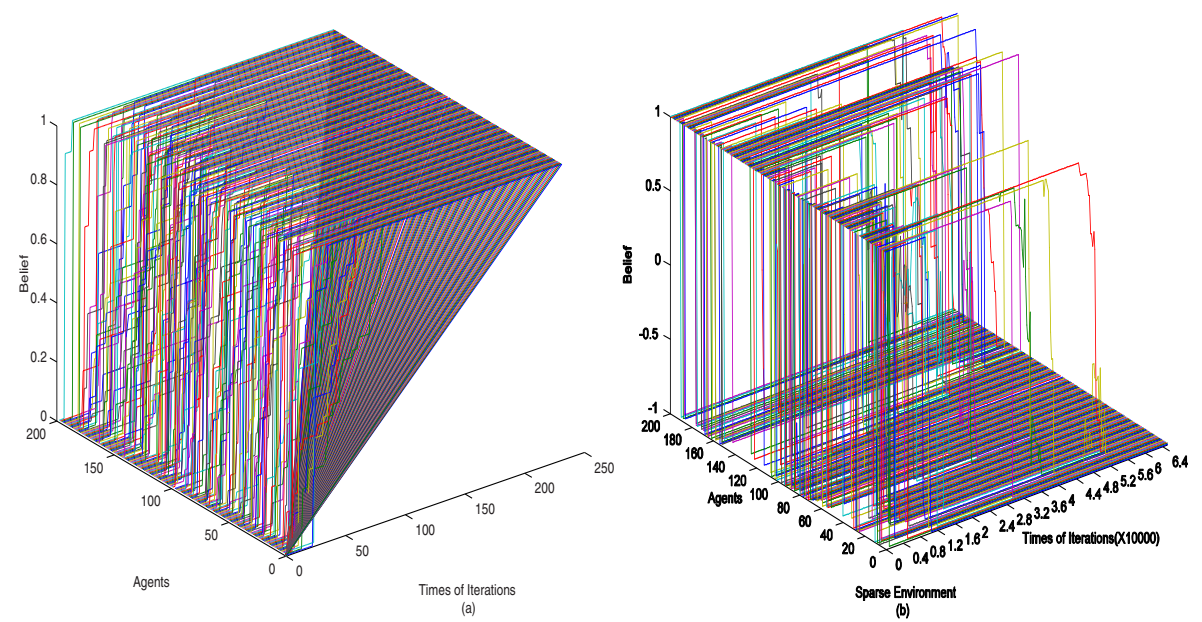

Fig. 3. Evolution of agent' belief in two kinds of environments :(a).Rumor environment. (b)Coexistence of rumor and anti-rumor environment.

There are plenty of more complex evolution paths except for aforementioned typical evolution paths in the coexistence of rumor and anti-rumor environment e.g., $1 \rightarrow-1 \rightarrow n \rightarrow p \rightarrow 1 \rightarrow p \rightarrow-1$. The above evolution path may be following situation. In the coexistence of rumor and anti-rumor environment, one agent changes from believing rumor to disbelieving rumor (believe anti-rumor) at one time when it hears anti-rumor information from neighboring agents. However, when the agent moves to another place, it may change slowly from believing anti-rumor information to believing rumor information at this time when neighboring agents of this agent are 
all to believe rumor information. If the agent moves to new place and meets neighboring agents that contain anti-rumor information, the agent may become to believe antirumor. Fig $3 b$ illustrates different evolution paths of agent for information in dense and sparse environment, respectively.

\section{Conclusion}

From economist point of view, rumors were often thought to be something rather irrational based on market behavior. However, from AI and mathematics (game theory) point of view, rumors were often thought to be rational because rumors are emerged by behavior of these agents who always try to maximize themselves payoff. In this paper, we used the framework of rumor game in order to investigate the emergence of social rumors and the efficiency of that process. In the framework, each agent can move randomly and interact with neighboring agents, and information is transmitted with word-of-mouth communication. We borrow ideas of reinforcement learning in order to capture the fact that agents use local update rules (i.e., to compute cumulative influence force (CIF)) to update their strategies. We make plenty of experiment to further discuss emergence of social rumor and anti-rumor.

\section{Acknowledgements}

This paper is based upon work supported by the JiangSu Province Science Technology Foundation under Grant No. BK2006567.

\section{References}

1. Cristiano Castelfranchi, modeling social action for AI agents, artificial intelligent, 103 (1998) 157-182.

2. Rosaria Conte, Social intelligence among autonomous agents, computational mathematical organization theory 5:3 (1999) 203-228

3. J Delgado, Emergence of social conventions in complex networks, Artificial Intelligence, 141 (2002) 171--185

4. PC Buzing, AE Eiben and MC Schut, merging communication and cooperation in evolving agent societies, Journal of Artificial Societies and Social Simulation,

5. Grant Michelson, Suchitra Mouly, Rumour and gossip in organisations: a conceptual study, Management Decision, Jun 2000 Volume: 38 Issue: 5 Page: 339 - 346.

6. Michael kosfeld, rumors and markets, journal of mathematical economics, 41 (2005) 646-664

7. Zanette, Damian H., Dynamics of rumor propagation on small-world networks, Phys. Rev. E 65, 041908 (2002)

8. Zanette, Damian H., Critical behavior of propagation on small-world networks, Phys. Rev. E 64, R050901 (2001)

9. D Fudenberg, J Tirole, Game theory, MIT Press Cambridge, Mass (1991) 